\title{
MicroRNA-451a plays a role in polycystic ovary syndrome by regulating ovarian granulosa cell proliferation and apoptosis
}

\author{
TIANJIN YANG ${ }^{1}$, LIE WANG $^{2}$, YUN ZHANG $^{3}$, JINDAN ZHENG $^{3}$ and LILI LIU ${ }^{3}$ \\ ${ }^{1}$ Department of Obstetrics and Gynecology, Jinzhou Medical University, Jinzhou, Liaoning 121000; \\ ${ }^{2}$ Reproductive Center, Qingdao Women and Children's Hospital, Qingdao, Shandong 266000; \\ ${ }^{3}$ Department of Obstetrics and Gynecology, The First Affiliated Hospital of Jinzhou \\ Medical University, Jinzhou, Liaoning 121000, P.R. China
}

Received May 7, 2020; Accepted September 15, 2020

DOI: $10.3892 /$ etm.2021.10015

\begin{abstract}
The present study aimed to investigate whether microRNA (miR)-451a plays a role in polycystic ovary syndrome by regulating the biological function of ovarian granulosa cells and investigate the underlying molecular mechanism. In the present study, reverse transcription-quantitative PCR (RT-qPCR) analysis detected markedly low expression of miR-451a in KGN cells. TargetScan predicted that cyclic AMP-dependent transcription factor ATF-2 (ATF2) was a potential target gene of miR-451a, which was confirmed by a Dual-Luciferase reporter gene assay. Moreover, western blotting and RT-qPCR experiments indicated that ATF2 was significantly overexpressed in KGN cells. In addition, western blotting and RT-qPCR experiments were utilized to assess cell transfection efficiency, and it was found that miR-451a mimic significantly increased miR-451a expression in KGN cells. Subsequently, MTT assay was performed to detect cell proliferation and flow cytometry was utilized to detect cell apoptosis. Western blot and RT-qPCR assays were utilized to assess the protein and mRNA expression of ATF2 and cyclin D1. The results confirmed that miR-451a mimic significantly decreased ATF2 protein and mRNA expression in KGN cells, and this decrease was reversed by ATF2-plasmid co-transfection. Moreover, miR-451a mimic inhibited cell proliferation, enhanced cell apoptosis, reduced cyclin D1 expression, increased caspase-3 activity and cleaved caspase-3 protein levels, while it reduced pro-caspase 3 protein levels in KGN cells, and these effects were significantly reversed by ATF2-plasmid. The present preliminary results demonstrated that miR-451a regulated the proliferation and apoptosis
\end{abstract}

Correspondence to: Dr Lili Liu, Department of Obstetrics and Gynecology, The First Affiliated Hospital of Jinzhou Medical University, No. 2, Section 5 Renmin Street, Guta, Jinzhou, Liaoning 121000, P.R. China

E-mail: i345633@163.com

Key words: microRNA-451a, polycystic ovary syndrome, ovarian granulosa cells, proliferation, apoptosis of ovarian granulosa cells by targeting ATF2. Thus, the miR-451a/ATF2 axis may be a new potential target for the treatment of polycystic ovary syndrome.

\section{Introduction}

Polycystic ovary syndrome (PCOS) is an endocrine disorder that causes health problems in $6-10 \%$ of premenopausal women (1). Hyperandrogenemia with chronic anovulation is the main phenotype of PCOS (2). The typical clinical manifestations of PCOS include acanthosis, alopecia, subfertility, acne vulgaris, obesity, seborrhea (3), hirsutism and menstrual disorders (4). PCOS is one of the main causes of anovulatory sterility in women (5). Furthermore, women with PCOS are at an increased risk of dyslipidemia, insulin resistance, type 2 diabetes and hypertension (6). However, although current research has provided more information on genetic background and the effect of environmental factors, the mechanism underlying the development of PCOS remains elusive.

During the development of ovarian follicles, it was observed that all stages of follicular atresia are associated with the apoptosis of granulosa cells (GCs). Based on this observation, GC apoptosis is considered to be the main mechanism underlying follicular atresia. A variety of factors have been shown to cause GC apoptosis, including depletion of cell survival factors (7). In addition, there is evidence that abnormal GC function may be the cause of abnormal follicle formation in PCOS (8). The high rate of apoptotic GCs is associated with low pregnancy rates, fertilization rates and embryonic dysplasia (9). However, the exact etiology of PCOS remains to be fully elucidated.

MicroRNAs (miRs/miRNAs) are highly conserved single-stranded non-coding RNA molecules composed of 20-24 nucleotides. miRNAs are important regulators of biological processes, such as cell proliferation, differentiation, migration and apoptosis (10). Dysregulation and differential expression of miRNAs have been associated with ovarian cancer, PCOS and uterus-related diseases. Furthermore, miRNAs have been found to be crucial for reproductive function, gonadal development and sex differentiation (11).

miR-451a is located on human chromosome 17q11.2. A previous study by Ibáñez et al suggested that one of the 
biomarkers for the diagnosis and treatment of PCOS during puberty may be circulating miR-451a (12). Low circulating levels of miR-451a in women with PCOS were previously reported, but its specific role remained unclear (13).

Although the pathogenic mechanism of PCOS must be further elucidated, previous findings indicated that the proliferation and survival of GCs may be implicated. The ovarian granulosa cell tumour cell line KGN has been widely used to investigate the biological function of ovarian granulosa cells in PCOS (14-16). Therefore, the aim of the present study was to determine whether miR-451a could regulate the biological function of KGN cells to verify its role in PCOS and to further explore the underlying molecular mechanisms, in order to provide new insights into the treatment of PCOS.

\section{Materials and methods}

Cell culture, treatment and transfection. Human ovarian granulosa cell tumour cell line KGN cells, normal ovarian surface epithelial IOSE80 cells and 293 cells were all purchased from the American Type Culture Collection. All cell lines were cultured in DMEM/F12 supplemented with 10\% FBS, $100 \mathrm{U} / \mathrm{ml}$ penicillin G (Life Technologies; Thermo Fisher Scientific, Inc.) and $0.1 \mathrm{mg} / \mathrm{ml}$ streptomycin sulfate (Gibco; Thermo Fisher Scientific, Inc.) in a humidified incubator at $37^{\circ} \mathrm{C}$ with $5 \% \mathrm{CO}_{2}$.

For the cell transfection experiments, vectors including miR-451a mimic, miR-451a mimic control, control-plasmid, ATF2-plasmid, miR-451a mimic + control-plasmid, or miR-451a mimic + ATF2-plasmid were transfected into KGN cells using Lipofectamine ${ }^{\mathrm{TM}} 2000$ (Life Technologies; Thermo Fisher Scientific, Inc.) according to the manufacturer's protocol.

Target prediction and luciferase reporter assay. TargetScan version 7.2 (www.targetscan.org) was used to analyze and predict potential targets for miR-451a. The 3'-untranslated region (3'-UTR) sequence of mutated (MUT) or wild-type (WT) ATF2, containing the putative miR-451a-binding site, was amplified by PCR and cloned into a psiCHECK vector (Promega Corporation). Site-directed mutagenesis using the QuikChange Lightning Site-Directed Mutagenesis kit (Agilent Technologies, Inc.) was performed according to the manufacturer's instructions to obtain the MUT 3'-UTR. Lipofectamine $^{\mathrm{TM}} 2000$ (Invitrogen; Thermo Fisher Scientific, Inc.) was used to co-transfect miR-451a mimic or mimic control with WT or MUT 3'-UTR vectors encoding Renilla luciferase into 293 cells. After 48 h, the activity of firefly luciferase was detected using the Dual-Luciferase Report Detection kit (Promega Corporation).

Determination of cell proliferation. MTT analysis was used to assess the proliferation of KGN cells. Briefly, KGN cells were seeded into 96 -well plates at a density of $5 \times 10^{3}$ cells/well. KGN cells were transfected with the vectors (mimic control, miR-451a mimic, miR-451a mimic + ATF2-plasmid or miR-451a mimic + control-plasmid) and incubated for $48 \mathrm{~h}$. KGN cells were incubated with $20 \mu \mathrm{l}$ MTT for $4 \mathrm{~h}$ at $37^{\circ} \mathrm{C}$. The cells were then lysed with $150 \mu 1 \mathrm{DMSO}$ for $10 \mathrm{~min}$ at room temperature at 24, 48, 72 and $96 \mathrm{~h}$ after transfection.

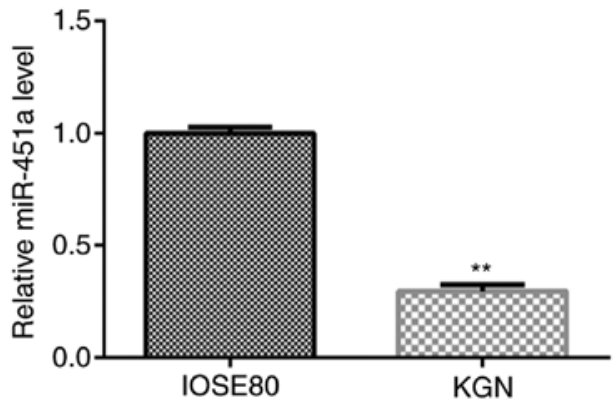

Figure 1. miR-451a expression in the IOSE80 and KGN cell lines. Reverse transcription-quantitative PCR analysis was performed to verify the expression of miR-451a in IOSE80 and KGN cells. ${ }^{* *} \mathrm{P}<0.01$ vs. IOSE80. miR, microRNA.

An absorption spectrophotometer (Olympus Corporation) was used to detect the optical density at a wavelength of $570 \mathrm{~nm}$.

Reverse transcription-quantitative PCR (RT-qPCR) analysis. The TRIzol one-step method (Invitrogen; Thermo Fisher Scientific, Inc.) was used to extract total RNA from KGN cells. Total RNA concentration was detected using a nucleic acid protein analyzer (Beckman Coulter, Inc.). RT was immediately performed using the Prime Script RT-PCR kit (Takara Biotechnology Co., Ltd.) according to the manufacturer's instructions to avoid RNA degradation. qPCR analysis was performed using the quantitative SYBR-Green PCR kit (Qiagen $\mathrm{GmbH}$ ) and the Mx4000 quantitative PCR system (Stratagene; Agilent Technologies, Inc.). The reaction conditions used for the qPCR were as follows: Initial denaturation for $5 \mathrm{~min}$ at $95^{\circ} \mathrm{C}$; followed by 40 cycles of denaturation at $95^{\circ} \mathrm{C}$ for $10 \mathrm{sec}$, annealing at $60^{\circ} \mathrm{C}$ for $30 \mathrm{sec}$ and extension at $72^{\circ} \mathrm{C}$ for $34 \mathrm{sec}$. The internal controls used were GAPDH or U6. Gene expression was analyzed using the $2^{-\Delta \Delta \mathrm{Cq}}$ method (17). The primer sequences for PCR were listed as follows: GAPDH forward, 5'-CTTTGGTATCGTGGAAGG ACTC-3' and reverse, 5'-GTAGAGGCAGGGATGATGTTC T-3'; U6 forward, 5'-GCTTCGGCAGCACATATACTAAAA T-3' and reverse, 5'-CGCTTCACGAATTTGCGTGTCAT-3'; miR-451a forward, 5'-ACACTCCAGCTGGGAAACCGT TACCATTAC-3' and reverse, 5'-CTCAACTGGTGTCGTGG AGTCGGCAATTCAGTTGAGCTTACAG-3; ATF2 forward, 5'-TACAAGTGGTCGTCGG-3' and reverse, 5'-CGGTTA CAGGGCAATC-3'; and cyclin D1 forward, 5'-CCGTCCATG CGGAAGATC-3 and reverse, 5'-GAAGACCTCCTCCTC GCACT-3'.

Flow cytometric analysis of apoptosis. $\mathrm{KGN}$ cells $\left(5 \times 10^{4}\right.$ cells per well) transfected with indicated plasmids or oligonucleotides were seeded in 6-well plates and incubated for $48 \mathrm{~h}$. For cell apoptosis analysis, the Annexin V-fluorescein isothiocyanate/propidium iodide Apoptosis Detection kit (Abcam) was used according to the manufacturer's instructions. Cell apoptosis was detected using the FACScan flow cytometry system equipped with CellQuest software version 5.1 (Becton, Dickinson and Company).

Caspase-3 activity detection. After transfection for $48 \mathrm{~h}$, KGN cells were collected through centrifugation $\left(600 \mathrm{x} \mathrm{g} ; 4^{\circ} \mathrm{C}\right.$; 
A

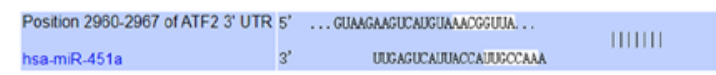

B

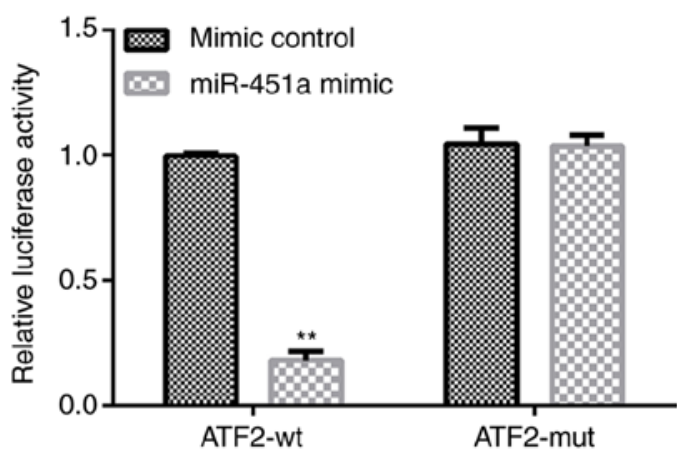

$\mathrm{D}$

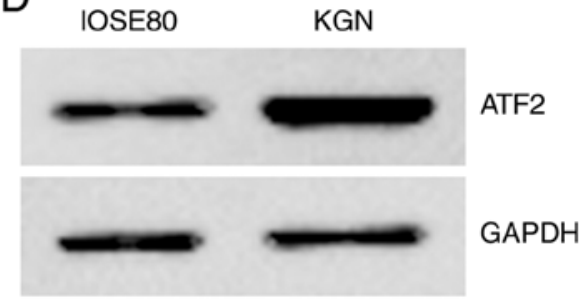

C

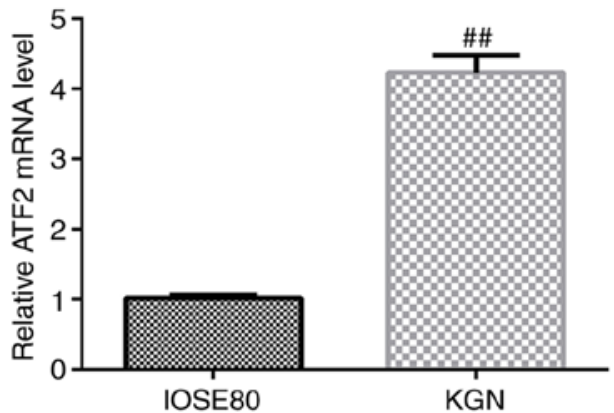

E

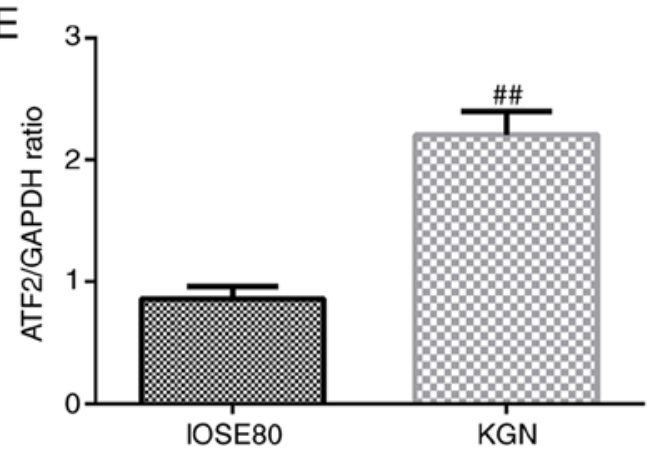

Figure 2. ATF2 is a target gene of miR-451a, which is significantly overexpressed in KGN cells. (A) Bioinformatics software (TargetScan) predicted that ATF2 was a potential target gene of miR-451a. (B) The Dual-Luciferase reporter gene system verified that ATF2 was a target gene of miR-451a. (C and D) Reverse transcription-quantitative PCR and western blot analyses were performed to verify the expression of ATF2 in IOSE80 and KGN cells. (E) The ATF2/GAPDH ratio was calculated. ${ }^{* *} \mathrm{P}<0.01$ vs. mimic control. ${ }^{\#} \mathrm{P}<0.01$ vs. IOSE80. ATF2, cyclic AMP-dependent transcription factor ATF-2; miR, microRNA.

5 min). Then the caspase-3 activity was immediately detected using a caspase-3 activity assay kit (cat no. C1116; Beyotime Institute of Biotechnology), following with the manufacturer's protocol.

Western blotting. Total protein was extracted from cells using $1 \mathrm{ml}$ RIPA cell lysis buffer (Sangon Biotech, Co., Ltd.). The proteins were quantified using a bicinchoninic acid protein assay reagent (Thermo Fisher Scientific, Inc.). A total of $40 \mu \mathrm{g}$ protein from each sample was separated by $10 \%$ SDS-PAGE, and subsequently transferred onto PVDF membranes (Bio-Rad Laboratories, Inc.). The membranes were blocked for $1 \mathrm{~h}$ in TBS with $0.1 \%$ Tween-20 in 5\% skimmed milk at room temperature, and incubated overnight at $4^{\circ} \mathrm{C}$ with the following primary antibodies: Anti-ATF2 (cat no. 35031; 1:1,000; Cell Signaling Technology, Inc.), anti-cyclin D1 (cat no. 55506; 1:10,000; Cell Signaling Technology, Inc.), anti-cleaved caspase-3 (cat no. ab32042; 1:500; Abcam), anti-pro-caspase-3 (cat no. ab3499; 1:500; Abcam) and anti-GAPDH (cat no. 5174; 1:1,000, Cell Signaling Technology, Inc.). This was followed by incubation with horseradish peroxidase-conjugated anti-rabbit IgG secondary antibody (cat no. 7074; 1:1,000; Cell Signaling Technology, Inc.) for $1 \mathrm{~h}$ at room temperature. Finally, enhanced chemiluminescence (EMD Millipore) was used to examine the immune complexes and the protein bands were quantified using ImageJ software (version 2.0; National Institutes of Health).
Statistical analysis. All experiments were performed three times. Data are expressed as the mean \pm SD and evaluated using Student's t-test or one-way ANOVA followed by Tukey's post hoc test. Data were analyzed using SPSS 22.0 software (IBM Corp.). $\mathrm{P}<0.05$ was considered to indicate a statistically significant difference.

\section{Results}

miR-451a expression in the IOSE80 and KGN cell lines. RT-qPCR analysis was performed to verify the expression of miR-451a in IOSE80 and KGN cells. As shown in Fig. 1, the expression of miR-451a in KGN cells was significantly lower compared with that in IOSE80 cells.

ATF2 is a target gene of miR-45la and is significantly overexpressed in KGN cells. Based on the target genes predicted by bioinformatics analysis, ATF2 was identified as a potential target of miR-451a (Fig. 2A). To confirm whether ATF2 is a target gene of miR-451a, a luciferase reporter assay was performed (Fig. 2B). Compared with the negative control, miR-451a mimic decreased WT 3'-UTR fluorescence (Fig. 2B), while MUT 3'-UTR was not altered following miR-451a transfection. Hence, the Dual-Luciferase reporter assay validated that ATF2 was a target gene of miR-45la.

To further verify the potential role of miR-451a in KGN cells, ATF 2 mRNA and protein expression levels were assessed 
A

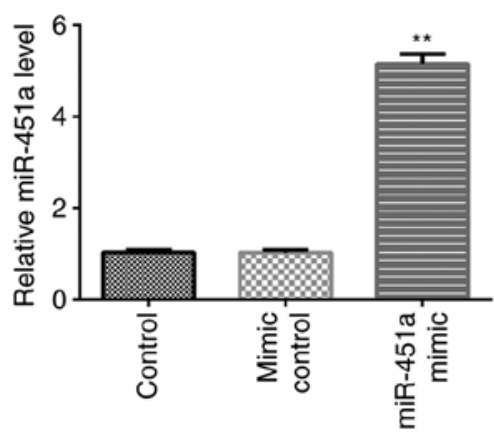

$\mathrm{B}$

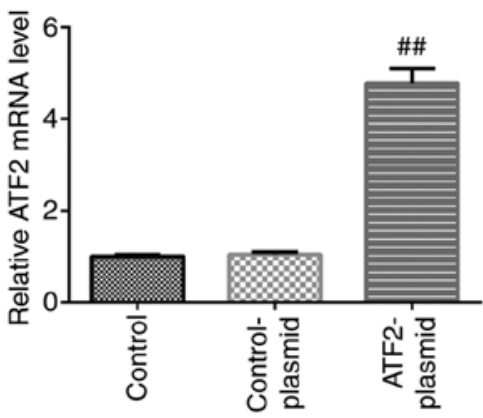

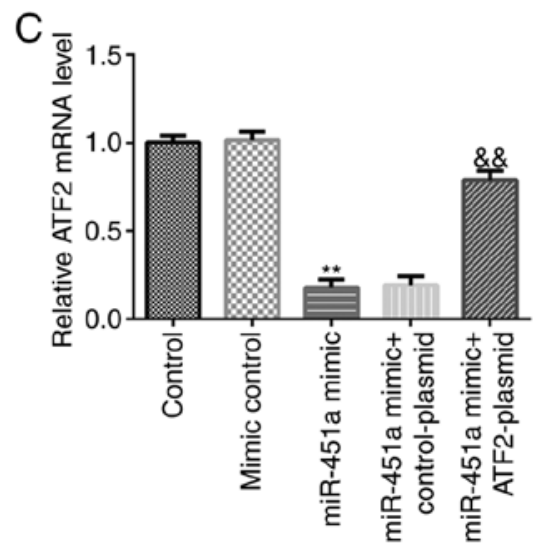
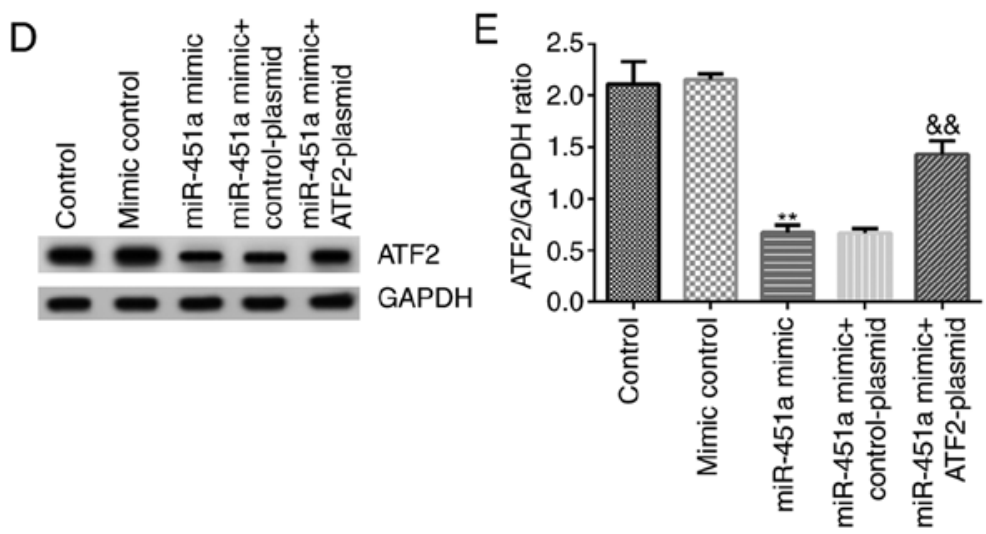

Figure 3. miR-451a negatively regulates ATF2 expression in KGN cells. (A) The miR-451a expression in KGN cells transfected with mimic control or miR-451a mimic was assessed using RT-qPCR analysis. (B) ATF2 mRNA expression in KGN cells transfected with control-plasmid or ATF2-plasmid was assessed using RT-qPCR analysis. (C and D) ATF2 mRNA and protein expression in KGN cells transfected with mimic control, miR-451a mimic, miR-451a mimic + control-plasmid or miR-451a mimic + ATF2-plasmid was detected using RT-qPCR and western blot assays. (E) The ATF2/GAPDH ratio was calculated. ${ }^{* *} \mathrm{P}<0.01$ vs. mimic control. ${ }^{\# \#} \mathrm{P}<0.01$ vs. control-plasmid. ${ }^{\&}{ }^{\&} \mathrm{P}<0.01$ vs. miR-451a mimic + control-plasmid. ATF2, cyclic AMP-dependent transcription factor ATF-2; miR, microRNA. RT-qPCR, reverse transcription-quantitative PCR.

in the KGN and IOSE80 cell lines. As shown in Fig. 2C-E, ATF2 expression was significantly increased in KGN cells compared with that in IOSE80 cells.

miR-451a inhibits KGN cell viability and induces apoptosis by reducing ATF2 expression. To determine the functional effects of miR-451a on KGN cell growth in vitro, KGN cells were transfected with mimic control, miR-451a mimic, ATF2-plasmid, control-plasmid, miR-451a mimic + control-plasmid or miR-451a mimic + ATF2-plasmid. RT-qPCR analysis was performed to confirm transfection efficiency. As shown in Fig. 3A, miR-451a mimic significantly increased miR-451a expression levels in KGN cells compared with the mimic control group. ATF2-plasmid significantly enhanced ATF2 mRNA expression in KGN cells compared with the control-plasmid group (Fig. 3B). Simultaneously, compared with the mimic control group, miR-451a mimic significantly decreased mRNA and protein expression of ATF2 in KGN cells, and this decrease was reversed by ATF2-plasmid co-transfection (Fig. 3C-E).

To further confirm the functional association between ATF2 and miR-451a, KGN cells were transfected with mimic control, miR-451a mimic, miR-451a mimic + control-plasmid or miR-451a mimic + ATF2-plasmid. Cell viability and apoptosis was evaluated by MTT and flow cytometry assays, respectively. As shown in Fig. 4A-C, miR-451a mimic significantly decreased KGN cell viability and induced apoptosis compared with the mimic control group, and these effects were significantly reversed by ATF2-plasmid co-transfection.

Western blotting and RT-qPCR analysis were performed to detect cyclin D1 protein and mRNA expression, respectively. As shown in Fig. 5A-C, compared with mimic controls, miR-451a mimic significantly reduced the expression of cyclin D1 at both the protein (Fig. 5A and B) and mRNA (Fig. 5C) levels in KGN cells, and these effects were significantly reversed by ATF2-plasmid co-transfection. Caspase-3 activity was assessed using a kit and the protein expression of cleaved caspase- 3 and pro-caspase- 3 was investigated by western blotting. The results demonstrated that miR-451a mimic significantly increased the activity of caspase-3 (Fig. 5D), enhanced cleaved caspase-3 protein levels, decreased the protein expression of pro-caspase-3 (Fig. 5E) and upregulated the cleaved caspase-3/pro-caspase -3 ratio (Fig. $5 \mathrm{~F}$ ) in KGN cells. In addition, these effects were notably reversed by ATF2-plasmid co-transfection. The results indicated that miR-451a inhibited KGN cell proliferation and induced apoptosis by reducing the expression of ATF2.

\section{Discussion}

The results of the present study demonstrated that miR-451a is downregulated in KGN cells and that miR-451a upregulation may inhibit GC proliferation, which may be the cause underlying the development of abnormal follicles in PCOS. The 

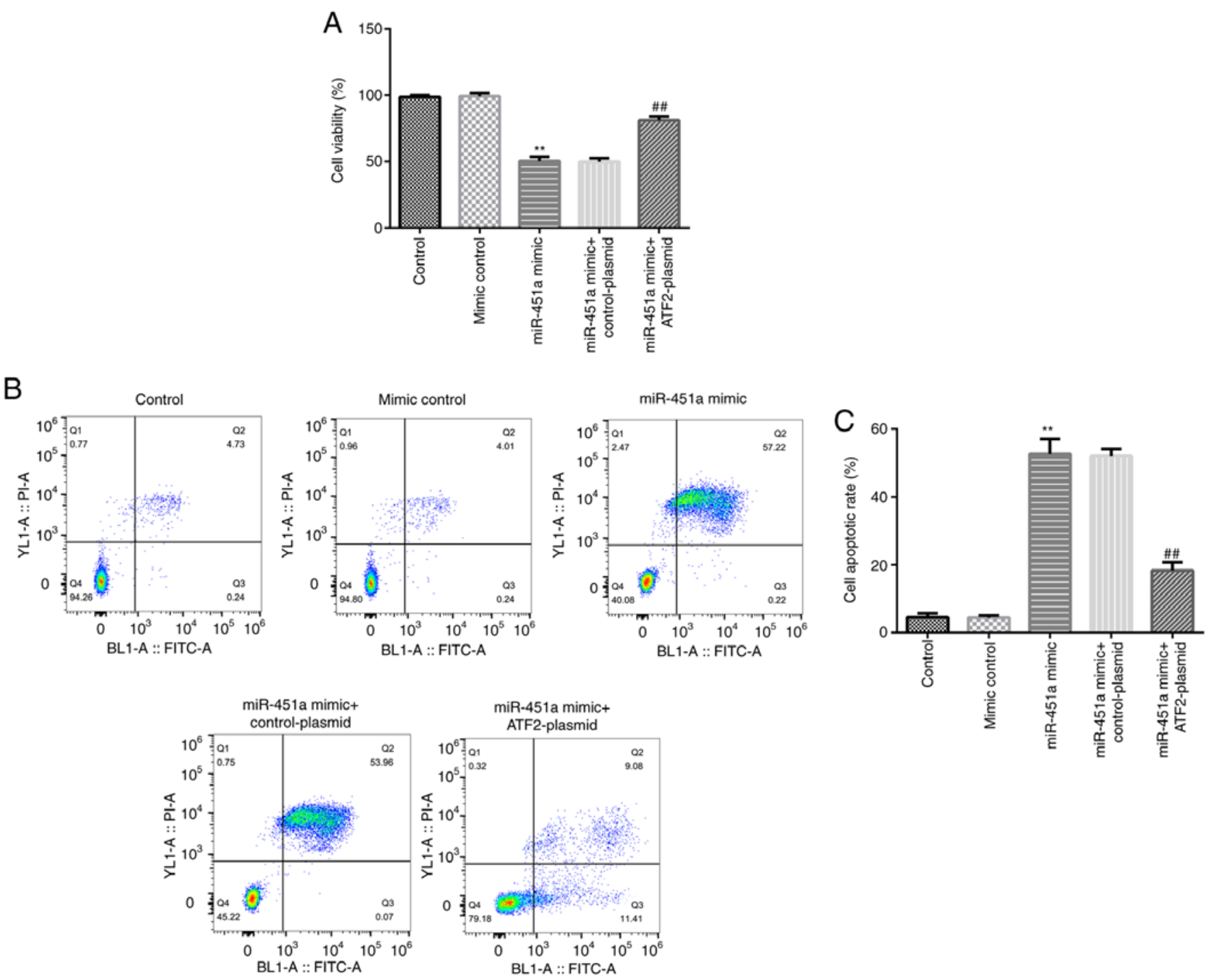

Figure 4. miR-451a inhibits KGN cell proliferation and induces apoptosis by reducing ATF2 expression. (A) MTT assay was performed to detect KGN cell viability. (B) Flow cytometry was performed to detect KGN cell apoptosis. (C) Cell apoptosis rates. ${ }^{* *} \mathrm{P}<0.01$ vs. mimic control. ${ }^{\# \#} \mathrm{P}<0.01 \mathrm{vs}$. miR-451a mimic + control-plasmid. ATF2, cyclic AMP-dependent transcription factor ATF-2; miR, microRNA.

expression of miR-451a was found to be significantly lower in KGN cells compared with that in IOSE80 cells. Based on previous studies, miR-451a is considered to play a role in the control of the cell proliferation, apoptosis and metastasis, by acting as a tumor suppressor (18-23). However, few studies have investigated the function of miR-451a in ovarian GCs. The present study determined that miR-451a may be a GC proliferation inhibitor.

Consistently with the results of a previous study (19), our further experiments revealed that ATF2 was a target of miR-451a. Based on Dual-Luciferase reporter gene assay results, miR-451a was shown to directly target the 3'-UTR of ATF2. Furthermore, transfection with miR-451a mimics reduced the protein and mRNA levels of ATF2 in KGN cells, whereas ATF2-plasmid transfection reversed these effects. These results indicated that miR-451a and its target ATF2 may jointly regulate the proliferation of GCs in PCOS.

ATF2 has been investigated in the context of several developmental and pathological conditions (24). Recent studies demonstrated that ATF2 may act as both a classic tumor suppressor (25) and as an oncogene (26). Moreover, it was reported that miR-451a suppressed cell migration and invasion in non-small cell lung cancer through targeting ATF2 (19). However, few studies have investigated ATF2 in ovarian GCs, and its mechanism of action remains unclear. ATF2 expression varies among different diseases. A previous study has shown that ATF2 is involved in the transcriptional regulation mechanism of mouse placental trophoblast giant cells and rat ovarian GCs in primary cultures (27). The present findings are consistent with these previous studies, whereby inhibition of ATF2 can inhibit GC proliferation.

The differences in miR-451a expression and cell proliferation observed in the present study may be attributed to differences in enzyme activity and protein and mRNA expression. The results of the present study demonstrated that miR-451a mimic significantly reduced the expression of cyclin D1 protein and mRNA in KGN cells, while it concurrently significantly increased the activity of caspase-3, increased cleaved caspase- 3 and reduced the protein expression of pro-caspase-3. These effects were significantly 
A

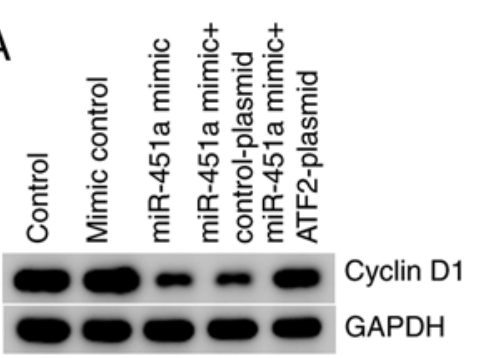

B

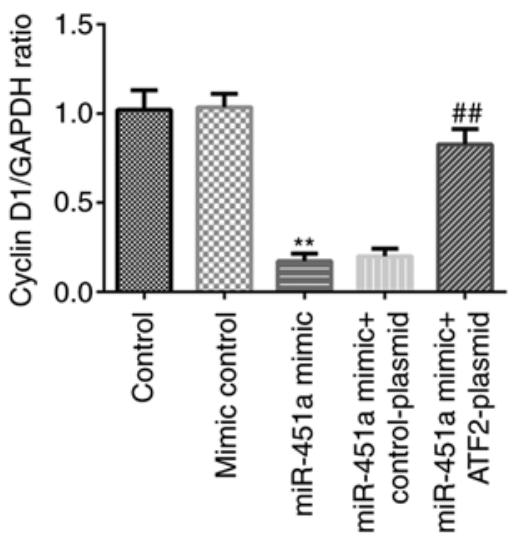

$\mathrm{D}$

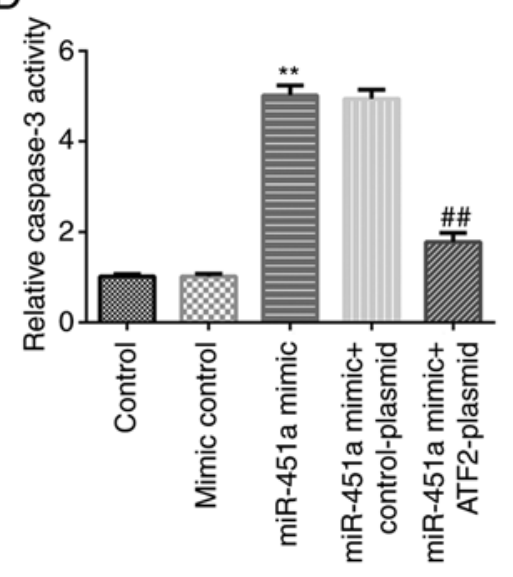

C

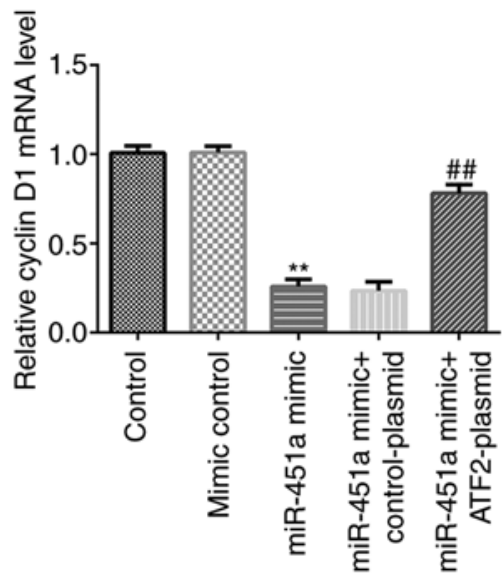

$\mathrm{F}$

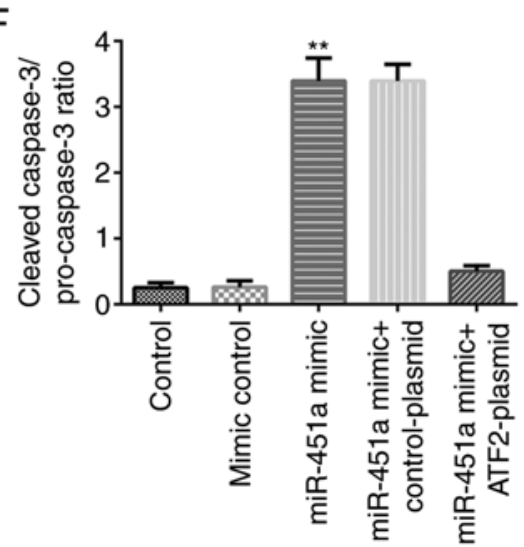

Figure 5. Effect of miR-451a expression on apoptosis-related gene/protein expression. (A-C) Western blotting and reverse transcription-quantitative PCR analysis were performed to detect cyclin D1 protein and mRNA expression, respectively. (D) A caspase-3 activity assay kit was used to detect the activity of caspase-3. (E) Cleaved caspase-3 and pro-caspase-3 protein expression were detected by western blotting. (F) The ratio of cleaved caspase-3/pro-caspase-3 was calculated. ${ }^{* *} \mathrm{P}<0.01$ vs. mimic control. ${ }^{\# \#} \mathrm{P}<0.01$ vs. miR-451a mimic + control-plasmid. miR, microRNA.

reversed by ATF2-plasmid co-transfection. Cyclin D1 is a key regulatory protein that promotes the transition through the restriction point in the G1 phase to $\mathrm{S}$ phase. Caspase-3 has been reported to be an important molecule in promoting various types of apoptosis (28). Its activation leads to the initiation of the caspase cascade, which is responsible for cell death (29). Zhen et al demonstrated that the regulatory mechanism of CCAAT/enhancer-binding protein (CEBP) in the cell cycle and steroid synthesis was inhibited in porcine ovaries. Additionally, the expression of cell cycle-related genes (cyclin D1, cyclin B1 and cyclin A1) was suppressed, indicating that knocking out the CEBP gene may inhibit apoptosis (30). The present results were consistent with those of previous studies. By targeting ATF2 in PCOS, miR-451a could decrease the expression of cyclin D1 and upregulate the activity and expression of cleaved caspase-3. However, the signaling pathway through which miR-451a/ATF2 regulate the proliferation and apoptosis of ovarian GCs remains largely unknown, and more in-depth investigation is required. Future research will focus on further exploring the molecular mechanisms underlying the regulatory effects of miR-451a/ATF2 on ovarian GCs and elucidating the related signaling pathways.

In summary, the results of the present study demonstrated that miR-451a regulated the proliferation and apoptosis of ovarian GCs by targeting ATF2, thereby providing novel insight into GC dysfunction in PCOS, and indicating miR-451a/ATF2 as a novel potential target for PCOS treatment.

\section{Acknowledgements}

Not applicable.

\section{Funding}

The present study was supported by the Guidance Program of the Natural Fund of Liaoning Provincial Science and Technology Department (grant no. 2019-ZD-0816).

\section{Availability of data and materials}

The datasets used and/or analyzed during the present study are available from the corresponding author on reasonable request.

\section{Authors' contributions}

TY contributed to the conception and design of the study and the manuscript preparation. LW, YZ and JZ contributed to the data acquisition, analysis and interpretation. LL contributed 
to the data acquisition and analysis. All authors read and approved the final manuscript.

\section{Ethics approval and consent to participate}

Not applicable.

\section{Patient consent for publication}

Not applicable.

\section{Competing interests}

The authors declare that they have no competing interests.

\section{References}

1. Doroszewska K, Milewicz T, Mrozińska S, Janeczko J, Rokicki R, Janeczko M, Warzecha D, Marianowski P: Blood pressure in postmenopausal women with a history of polycystic ovary syndrome. Prz Menopauzalny 18: 94-98, 2019.

2. Seyam E, Al Gelany S, Abd Al Ghaney A, Mohamed MAA, Youseff AM, Ibrahim EM, Khalifa EM and Hefzy E: Evaluation of prolonged use of statins on the clinical and biochemical abnormalities and ovulation dysfunction in single young women with polycystic ovary syndrome. Gynecolo Endocrinol 34: 589-596, 2018.

3. Sharma S, Mathur DK, Paliwal V and Bhargava P: Efficacy of metformin in the treatment of acne in women with polycystic ovarian syndrome: A newer approach to acne therapy. J Clin Aes DermatO 12: 34-38, 2019.

4. Moini Jazani A, Nasimi Doost Azgomi H, Nasimi Doost Azgomi A and Nasimi Doost Azgomi R: A comprehensive review of clinical studies with herbal medicine on polycystic ovary syndrome (PCOS). Daru 27: 863-877, 2019.

5. Kriedt KJ, Alchami A and Davies MC: PCOS: Diagnosis and management of related infertility. Obstet Gynaecol Reprod Med 29: 1-5, 2019

6. ArentzS, AbbottJA,SmithCA and Bensoussan A:Herbalmedicine for the management of polycystic ovary syndrome (PCOS) and associated oligo/amenorrhoea and hyperandrogenism; a review of the laboratory evidence for effects with corroborative clinical findings. BMC Complement Altern Med 14: 511, 2014.

7. Yu YS, Sui HS, Han ZB, Li W, Luo MJ and Tan JH: Apoptosis in granulosa cells during follicular atresia: Relationship with steroids and insulin-like growth factors. Cell Res 14: 341-346, 2014.

8. Niu Z, Ye Y, Xia L, Feng Y and Zhang A: Follicular fluid cytokine composition and oocyte quality of polycystic ovary syndrome patients with metabolic syndrome undergoing in vitro fertilization. Cytokine 91: 180-186, 2017.

9. Salei N, Hellberg L, Köhl J and Laskay T: Enhanced survival of Leishmania major in neutrophil granulocytes in the presence of apoptotic cells. PLoS One 12: e0171850, 2017.

10. Xiang Y, Song Y, Li Y, Zhao D, Ma L and Tan L: miR-483 is down-regulated in polycystic ovarian syndrome and inhibits KGN cell proliferation via targeting insulin-like growth factor 1 (IGF1). Med Sci Monit 22: 3383-3393, 2016.

11. Sun L, Zuo Z, Luo H, Chen M, Zhong Y, Chen Y and Wang C: Chronic exposure to phenanthrene influences the spermatogenesis of male sebastiscus marmoratus: U-shaped effects and the reason for them. Environ Sci Technol 45: 10212-10218, 2011.

12. Ibáñez L, Oberfield SE, Witchel S, Auchus RJ, Chang RJ, Codner E, Dabadghao P, Darendeliler F, Elbarbary NS, Gambineri A, et al: An international consortium update: Pathophysiology, diagnosis, and treatment of polycystic ovarian syndrome in adolescence. Horm Res Paediatr 88: 371-395, 2017.

13. Díaz M, Bassols J, López-Bermejo A, de Zegher F and Ibáñez L: Low circulating levels of miR-451a in girls with polycystic ovary syndrome: Different effects of randomized treatments. J Clin Endocri Metabo 105: dgz204, 2020.
14. Sun X, Su S, Zhang G, Zhang H and Yu X: MiR-204 suppresses cell proliferation and promotes apoptosis in ovarian granulosa cells via targeting TPT1 in polycystic ovary syndrome. Biochem Cell Biol 97: 554-562, 2019.

15. He T, Sun Y, Zhang Y, Zhao S, Zheng Y, Hao G and Shi Y: MicroRNA-200b and microRNA-200c are up-regulated in PCOS granulosa cell and inhibit KGN cell proliferation via targeting PTEN. Reprod Biol Endocrinol 17: 68, 2019.

16. Li Y, Liu YD, Zhou XY, Chen SL, Chen X, Zhe J, Zhang J, Zhang QY and Chen YX: MiR-29a regulates the proliferation, aromatase expression, and estradiol biosynthesis of human granulosa cells in polycystic ovary syndrome. Mol Cell Endocrinol 498: 110540, 2019.

17. Livak KJ and Schmittgen TD: Analysis of relative gene expression data using real-time quantitative PCR and the 2(-Delta Delta C(T)) method. Methods 25: 402-408, 2001

18. Minna E, Romeo P, Dugo M, De Cecco L, Todoerti K, Pilotti S, Perrone F, Seregni E, Agnelli L, Neri A, et al: miR-451a is underexpressed and targets AKT/mTOR pathway in papillary thyroid carcinoma. Oncotarget 7: 12731-12747, 2016.

19. Shen YY, Cui JY, Yuan J and Wang X: MiR-451a suppressed cell migration and invasion in non-small cell lung cancer through targeting ATF2. Eur Rev Med Pharmacol Sci 22: 5554-5561, 2018.

20. Zhao S, Li J, Zhang G, Wang Q, Wu C, Zhang Q, Wang H, Sun P, Xiang R and Yang S: Exosomal miR-451a functions as a tumor suppressor in hepatocellular carcinoma by targeting LPIN1. Cell Physiol Biochem 53: 19-35, 2019.

21. Xu K, Han B, Bai Y, Ma XY, Ji ZN, Xiong Y, Miao SK, Zhang YY and Zhou LM: MiR-451a suppressing BAP31 can inhibit proliferation and increase apoptosis through inducing ER stress in colorectal cancer. Cell Death Dis 10: 152, 2019.

22. Wei GY, Hu M, Zhao L and Guo WS: MiR-451a suppresses cell proliferation, metastasis and EMT via targeting YWHAZ in hepatocellular carcinoma. Eur Rev Med Pharmacol Sci 23: 5158-5167, 2019.

23. Li M, Song Q, Li H, Lou Y and Wang L: Circulating miR-25-3p and miR-451a may be potential biomarkers for the diagnosis of papillary thyroid carcinoma. PLoS One 10: e0132403, 2015.

24. Watson G, Ronai ZA and Lau E: ATF2, a paradigm of the multifaceted regulation of transcription factors in biology and disease. Pharmacol Res 119: 347-357, 2017.

25. Dai Y, Zang Y, Li J, Liu Y and Wan B: miR-181a and miR-203 inhibit migration and invasion of laryngeal carcinoma cells by interacting with ATF2. Int J Clin Exp Pathol 12: 133-141, 2019.

26. Li Q, Gao WQ, Dai WY, Yu C, Zhu RY and Jin J: ATF2 translation is induced under chemotherapeutic drug-mediated cellular stress via an IRES-dependent mechanism in human hepatic cancer Bel7402 cells. Oncol Lett 12: 4795-4802, 2016.

27. Yivgi-Ohana N, Sher N, Melamed-Book N, Eimerl S, Koler M, Manna PR, Stocco DM and Orly J: Transcription of steroidogenic acute regulatory protein in the rodent ovary and placenta: Alternative modes of cyclic adenosine 3', 5'-monophosphate dependent and independent regulation. Endocrinology 150: 977-989, 2009

28. Riaz H, Liang A, Khan MK, Dong P, Han L, Shahzad M, Chong Z, Ahmad S, Hua G and Yang L: Somatostatin and its receptors: Functional regulation in the development of mice Sertoli cells. J Steroid Biochem Mol Biol 138: 257-266, 2013.

29. Han L, Wu C, Riaz H, Bai L, Chen J, Zhen Y, Guo A and Yang L: Characterization of the mechanism of inhibin $\alpha$-subunit gene in mouse anterior pituitary cells by RNA interference. PLoS One 8: e74596, 2013.

30. Zhen YH, Wang L, Riaz H, Wu JB, Yuan YF, Han L, Wang YL, Zhao Y, Dan Y and Huo LJ: Knockdown of CEBP $\beta$ by RNAi in porcine granulosa cells resulted in $\mathrm{S}$ phase cell cycle arrest and decreased progesterone and estradiol synthesis. J Steroid Biochem Mol Biol 143: 90-98, 2014.

This work is licensed under a Creative Commons Attribution-NonCommercial-NoDerivatives 4.0 International (CC BY-NC-ND 4.0) License. 